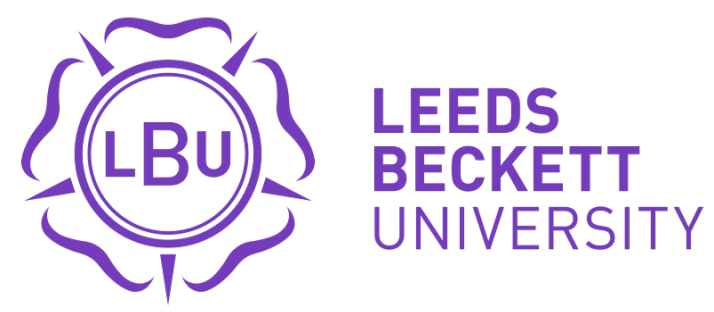

Citation:

Hobkirk, JP and King, RF and Gately, P and Pemberton, P and Smith, A and Barth, JH and Carroll, S (2012) Longitudinal factor analysis reveals a distinct clustering of cardiometabolic improvements during intensive, short-term dietary and exercise intervention in obese children and adolescents. Metabolic syndrome and related disorders, 10 (1). 20 - 25. ISSN 1540-4196 DOI: https://doi.org/10.1089/met.2011.0050

Link to Leeds Beckett Repository record:

https://eprints.leedsbeckett.ac.uk/id/eprint/267/

Document Version:

Article (Accepted Version)

The aim of the Leeds Beckett Repository is to provide open access to our research, as required by funder policies and permitted by publishers and copyright law.

The Leeds Beckett repository holds a wide range of publications, each of which has been checked for copyright and the relevant embargo period has been applied by the Research Services team.

We operate on a standard take-down policy. If you are the author or publisher of an output and you would like it removed from the repository, please contact us and we will investigate on a case-by-case basis.

Each thesis in the repository has been cleared where necessary by the author for third party copyright. If you would like a thesis to be removed from the repository or believe there is an issue with copyright, please contact us on openaccess@leedsbeckett.ac.uk and we will investigate on a case-by-case basis. 


\title{
Longitudinal Factor Analysis Reveals a Distinct Clustering of Cardiometabolic Improvements During Intensive, Short-Term Dietary and Exercise Intervention in Obese Children and Adolescents
}

\author{
James P. Hobkirk, B.Sc., ${ }^{1,2}$ Roderick F. King, Ph.D., ${ }^{2}$ Paul Gately, Ph.D., ${ }^{2}$ Philip Pemberton, M.Sc., ${ }^{3}$ \\ Alexander Smith, Ph.D., ${ }^{3}$ Julian H. Barth, M.D., ${ }^{4}$ and Sean Carroll, Ph.D. ${ }^{2}$
}

\begin{abstract}
Objective: The aim of this study was to investigate changes in cardiometabolic clustering characteristics in response to highly significant weight loss.

Background: Pre-post analysis of a lifestyle intervention for the treatment of obesity and the assessment of interrelated metabolic changes were analyzed using principal component analysis (PCA). A total of $n=75$ clinically obese boys and girls [standardized body mass index (sBMI) 3.07 \pm 0.59 ] aged 8-18 years were assessed after lifestyle intervention ( $30 \pm 12$ days).

Results: There were favorable improvements in BMI waist circumference, fasting insulin, triglycerides (TGs), systolic blood pressure (SBP) and diastolic blood pressure (DBP) (all $P<0.001$ ). PCA was performed using a simple conceptual model of changes in six metabolic variables: Overall and central obesity (BMI and waist circumference), dyslipidemia [TG and high-density lipoprotein cholesterol (HDL-C)], insulin resistance [fasting insulin or homeostasis model assessment of insulin resistance (HOMA-IR)], and blood pressure [SBP or mean arterial pressure (MAP)]. PCA models consistently identified two factors underlying the changes in six cardiometabolic variables. These were labeled a "metabolic" factor, typically including waist circumference, fasting triglyceride, insulin, or HOMA-IR and HDL-C (negatively) and an "obesity/blood pressure" factor, typically loading waist, BMI, SBP or MAP, and occasionally fasting insulin/HOMA-IR). The metabolic and obesity/blood pressure factors explained $26.5 \%-28.4 \%$ and $30.4 \%-31.9 \%$, of the variance in metabolic risk factors changes, respectively. Reductions in BMI, waist circumference, and HOMA-IR (or fasting insulin) were central underlying features of cardiometabolic changes.

Conclusion: There were significant and favorable cardiometabolic risk factor changes to short-term weight-loss. A distinct clustering of cardiometabolic responses supports the etiological importance of both overall and central obesity and insulin resistance in the modification of cardiometabolic risk in obese youths.
\end{abstract}

\section{Introduction}

TNSUlin RESISTANCE AND HYPERINSULINEMIA were initially I proposed as the important underlying construct associated with cardiometabolic clustering. ${ }^{1}$ Subsequently, abdominal obesity has been strongly linked to fasting hyperinsulinemia, atherogenic dyslipidemia [reduced high density lipoprotein cholesterol (HDL-C), hypertriglyceridemia] and raised blood pressure. ${ }^{2}$ Cardiometabolic clus- tering is highly prevalent among obese children and adolescents. ${ }^{3}$ However, the usefulness of the diagnostic term "metabolic syndrome" has been widely debated. ${ }^{4}$ The construct of cardiometabolic risk is complicated by its physiological complexity and high degree of intercorrelation among cardiometabolic variables. ${ }^{5}$ Exploratory factor analysis (EFA), a multivariate statistical data reduction technique, has been increasingly applied to summarize information from cardiometabolic variables to assess

${ }^{1}$ Department of Academic Cardiology, Castle Hill Hospital, Hull, United Kingdom.

${ }^{2}$ Carnegie Research Institute, Leeds Metropolitan University, Leeds, United Kingdom.

${ }^{3}$ Specialist Assay Laboratory, Clinical Biochemistry, Manchester Royal Infirmary, Manchester, United Kingdom.

${ }^{4}$ Clinical Biochemistry, Leeds General Infirmary, Leeds, United Kingdom. 
cumulative cardiometabolic risk ${ }^{6}$ or specifically to examine the structure underlying the metabolic syndrome components within obese pediatric cohorts. ${ }^{7}$

However, only one study has used factor analysis techniques to examine prospective changes in cardiometabolic risk factors. ${ }^{8}$ Previous studies have shown the robust nature of the linear relationships among derived "factors" over time within adolescents and emphasized its potential for assessing information about causal pathways within cardiometabolic clustering compared with cross-sectional analyses. ${ }^{6}$ Long-term weight-loss reflected by sBMI changes, was tightly linked to changes in surrogate measures of insulin sensitivity and several cardiometabolic variables among obese adolescents. ${ }^{9}$ To the best of our knowledge, no exploratory factor analysis has investigated the dynamic changes in cardiometabolic risk factors in clinically obese children and adolescents who have lost weight through an intensive exercise and dietary intervention. The aim of the present study was to examine of the pattern of change in cardiometabolic risk factors induced by a short-term intensive residential lifestyle intervention. The analytical structure of cardiometabolic clustering in obese children and adolescents utilizing different metabolic variable models will be explored.

\section{Methods}

The obese children and adolescents were attendees of the Carnegie International Camp (CIC), a multifactorial residential summer weight loss program. This analysis includes 75 first-time attendees of the CIC who volunteered for blood sampling and completed both pre- and post intervention blood sampling. Data from 3 consecutive years (2004-2006) was used. Mainly British children and adolescents (8-18 years) attended and were recruited via self-parental referral, medical and social service referral, or educational organizations. Acceptance onto the weight-loss program was reliant on having a BMI $>85^{\text {th }}$ percentile. ${ }^{10}$ There were no other inclusion criteria. The subjects were primarily Caucasians $(n=72$, Asian $n=1$, and black $n=2)$. Tanner development stages were not assessed.

Details of the program are reported elsewhere. ${ }^{11}$ Briefly the CIC program incorporated dietary restriction, physical activity/exercise, and lifestyle education. The physical activity combined structured fun, skill-based activities consisting of six daily $1-\mathrm{h}$ sessions. The dietary intervention provided sufficient daily energy to meet the individual's basal requirements adjusted for age and body mass. Energy intake $\left(\mathrm{kcal} \cdot \mathrm{day}^{-1}\right)$ was provided as three meals and a snack each day. Each child was assigned to one of four diet groups ranging from 1,050 to $3,050 \mathrm{kcal} \cdot \mathrm{day}^{-1}$ based upon their age and body mass. The diet consisted of $15 \%$ protein, $30 \%-35 \%$ fat, and $50 \%-55 \%$ carbohydrate. A registered dietitian designed the diets. Each child underwent four 1-h obesity-related lifestyle educational sessions per week. Ethical approval was granted by Leeds West National Health Service Research Ethics Committee, Leeds, UK. All children and parents provided informed written assent/consent to participate.

\section{Anthropometric and body composition measures}

Bioelectrical impedance analysis (BLA) assessed body mass using a Tanita TBF-310 (Tanita Corp, Tokyo, Japan). Height was measured to the nearest $0.1 \mathrm{~cm}$ using a floor-standing
Seca stadiometer (model 220). BMI was calculated (weight/ height ${ }^{2}$ ), and sBMI was calculated using the cross-sectional stature and weight reference curves for the UK Waist was assessed at the midpoint (narrowest point between the lowest rib and the iliac crest) to the nearest $0.1 \mathrm{~cm}$ using an inflexible tape measure. Blood pressure was measured using a mercury sphygmomanometer (Mercurial BK1001) on the left arm. All subjects sat quietly for $5 \mathrm{~min}$ prior to measurement. A single measurement was taken in the semisupine position. In a subsample of participants (2006 cohort), a digital blood pressure monitoring device as opposed to manual method was used (Omron HEM-773AC, UK). Measurement protocols defined the appropriate cuff size to be used in all measurements.

\section{Biochemical measurements}

Fasting blood samples (12-13.5h) were drawn by venipuncture. Analyses for lipoprotein-lipids, glucose, and insulin were undertaken using routine clinical analyses within the Department of Clinical Biochemistry, Leeds General Infirmary, UK. Serum HDL-C was determined by an elimination and catalase procedure. Fasting insulin concentration was analyzed by an ADVIA sandwich immunoassay using chemiluminescent technology. The intraassay coefficient of variation for plasma insulin was $3.5 \%$. Plasma apolipoprotein B (apo B) was measured by an enzyme-linked immunosorbent assay (ELISA) technique. The within-batch coefficient of variation for apoB was $8.7 \%$ and the between-batch coefficient of variation was $9.9 \%$ and was measured in the Specialist Assay Laboratory, Clinical Biochemistry, Manchester Royal Infirmary.

\section{Statistical analysis}

All variables were assessed for normality of distribution. Paired-sample $t$-tests were used to determine mean differences for pre-post intervention metabolic variables. Pearson correlation coefficients were used to assess the relationships between continuous variables. PCA was applied to longitudinal changes in cardiometabolic variables. The four EFA models evaluated were distinguished by the main components of the metabolic syndrome. This included, overall and central obesity measures (BMI, waist circumference), dyslipidemia (TGs and HDL-C), and a single measure of blood pressure (systolic or mean arterial pressure) and marker of insulin resistance [fasting insulin or homeostasis model assessment (HOMA)]. All models included BMI and waist circumference, combined with four other metabolic variables. These included two lipoprotein-lipid markers (TG, HDL-C), one insulin resistance (IR) marker (fasting insulin or HOMA-IR), and one blood pressure component [systolic blood pressure (SBP) or mean arterial blood pressure (MAP)]. EFA involves three procedures: (1) Extraction of factors; (2) rotation of factors to facilitate interpretation of the latent factors identified; and (3) naming and interpretation of each underlying factor based on the estimated values for the factor loadings. ${ }^{12}$

The Kaiser-Meyer-Olkin (KMO) statistic was used as a measure of sampling adequacy, and the Bartlett test of sphericity was used as a measure of the ability to perform a factor analysis. ${ }^{5}$ The KMO statistic varies from 0 to 1.0 , and an overall KMO of 0.60 or higher (preferably above 0.7 ) indicates suitability of the data for factor analysis based on the correlations and partial correlations among metabolic variables. Furthermore, communality statistics were examined to 
assess the 'reliability' of the metabolic risk variables in each analysis. PCA transforms the original variables into 'factors' that are uncorrelated and account for decreasing proportions of the variance of the data. Factors with a variance or eigenvalue $>1.0$ were retained for inspection.

Previously, the process of factor rotation and selection of thresholds for factor loading has been arbitrary, but may importantly affect the results and their interpretation. ${ }^{12}$ Accordingly, we compared different rotational techniques to facilitate the interpretation of factors. We used the orthogonal varimax rotation method. However, direct oblimin, an oblique rotation technique, was initially used in all analyses as a filter technique. Following oblique rotation, the factor (component) correlation matrix was examined. Small correlations amongst identified factors $(<0.32$, corresponding to $10 \%$ explained variance) were considered as supporting orthogonality in the model. Each factor was characterized by its loading or correlation with the original metabolic variables, which, in this study, included the metabolic changes over time. Factor loadings $\geq 0.4$ were retained and interpreted as the contribution of that metabolic variable to the specific factor. Conventionally, variables that have a factor loading of 0.4 or greater are considered to load on a factor. Variables were considered to have potential loading if correlation coefficients were $>0.3$. All analyses were conducted using SPSS version 17.0 (Chicago, IL).

\section{Results}

The lifestyle intervention lasted a mean of $30 \pm 12$ days (range 45 days), and produced a significant loss of body mass, BMI, waist circumference, total cholesterol (TC), and low-density lipoprotein cholesterol (LDL-C), HDL-C, TG, insulin, glucose, HOMA-IR, and SBP and DBP (all $P<0.001$; see Table 1). Pearson correlations showed that changes in measures of obesity (BMI and waist) were significantly (all $P<0.05)$ positively associated with changes in SBP or MAP (all $r=0.32-0.37$ ). Changes in waist circumference were more strongly related to changes in fasting insulin and HOMA-IR $(r=0.43$ and 0.44 , respectively) than BMI (both $r=0.29$ ).
Waist circumference correlated positively with TGs $(r=0.34$, $P<0.05$ ). Changes in fasting insulin concentration and HOMA-IR correlated positively with changes in TGs $(r=0.43, P<0.01)$. Blood pressure measures were not significantly related to changes in markers of insulin resistance. In all EFA undertaken with six metabolic variables, the Bartlett test of sphericity was significant (chi-squared values ranging from approximately $\chi^{2}=71.6, P<0.001$ to $\chi^{2}=78.0, P<0.001$, respectively). The overall KMO statistic (a measure of sampling adequacy) was consistent at 0.7 , indicating good suitability of the longitudinal change data for factor analysis. Four separate PCAs were undertaken, entering six metabolic variables into exploratory PCA models (Table 2). These models consistently identified two underlying factors and explained approximately $58 \%$ of the data variance. Preliminary oblique factor rotations showed correlations of less than \pm 0.26 between the two factors identified, indicating suitability of orthogonal rotation to produce two unrelated factors.

The first uncorrelated factor was characterized by positive correlation coefficients (loadings $<0.75$ ) with waist circumference and BMI with either SBP or MAP and, in several analyses, a lower loading of fasting insulin or HOMA-IR (correlation coefficients of $>0.3-0.40$ ). We labeled this factor as "obesity/blood pressure." The second factor was characterized as a "metabolic" factor, where changes in the lipid factors (TG $>0.8$ and negatively HDL-C greater than -0.5 ) were correlated with changes in central obesity (WC, $>0.3-$ 0.4 ) and fasting insulin or HOMA-IR (correlation coefficients of $>0.50$ ). Notably, within three of the four EFAs entering six variables, waist circumference and a surrogate measure of insulin resistance (fasting insulin or HOMA-IR) loaded together on both underlying factors (Table 2).

\section{Discussion}

The present study is the first to report longitudinal changes in cardiometabolic risk factors and their clustering characteristics in obese children and adolescents during an intensive residential weight loss intervention. Previous

Table 1. Anthropometric, Body Composttion, and Cardiometabolic Changes Following Acute Weight Loss in Obese Children and Adolescents

\begin{tabular}{lcccc}
\hline & $\mathrm{n}$ & Pre Mean $\pm S D$ & Post Mean $\pm S D$ & P value \\
\hline Body mass $(\mathrm{kg})$ & 75 & $94.2 \pm 22.1$ & $88.8 \pm 20.7$ & $<0.001$ \\
BMI $\left(\mathrm{kg} \cdot \mathrm{m}^{-2}\right)$ & 75 & $34.2 \pm 6.4$ & $32.1 \pm 6.0$ & $<0.001$ \\
sBMI & 75 & $3.07 \pm 0.59$ & $2.82 \pm 0.63$ & $<0.001$ \\
Waist circumference $(\mathrm{cm})$ & 73 & $98.4 \pm 12.0$ & $92.9 \pm 10.7$ & $<0.001$ \\
SBP $(\mathrm{mmHg})$ & 73 & $120 \pm 13$ & $116 \pm 11$ & $<0.01$ \\
DBP $(\mathrm{mmHg})$ & 72 & $72 \pm 10$ & $68 \pm 10$ & $<0.05$ \\
TC $\left(\mathrm{mmol} \cdot \mathrm{L}^{-1}\right)$ & 75 & $4.15 \pm 0.69$ & $3.27 \pm 0.58$ & $<0.001$ \\
LDL-C $\left(\mathrm{mmol} \cdot \mathrm{L}^{-1}\right)$ & 75 & $2.48 \pm 0.57$ & $1.80 \pm 0.45$ & $<0.001$ \\
HDL-C $\left(\mathrm{mmol} \cdot \mathrm{L}^{-1}\right)$ & 75 & $1.20 \pm 0.24$ & $1.11 \pm 0.23$ & $<0.001$ \\
TC/HDL-C ratio & 75 & $3.55 \pm 0.74$ & $2.99 \pm 0.60$ & $<0.001$ \\
TG (mmol $\left.\mathrm{L}^{-1}\right)$ & 75 & $1.11 \pm 0.49$ & $0.84 \pm 0.31$ & $<0.001$ \\
Apo B $\left(\mathrm{mg} \cdot \mathrm{dL}^{-1}\right)$ & 73 & $89.3 \pm 21.8$ & $69.3 \pm 16.9$ & $<0.001$ \\
Glucose $\left(\mathrm{mmol} \cdot \mathrm{L}^{-1}\right)$ & 74 & $4.79 \pm 0.40$ & $4.55 \pm 0.31$ & $<0.001$ \\
Insulin $\left(\mathrm{mU} \cdot \mathrm{L}^{-1}\right)$ & 74 & $17.5 \pm 11.1$ & $12.6 \pm 7.7$ & $<0.001$ \\
HOMA-IR & 73 & $3.7 \pm 2.5$ & $2.6 \pm 1.7$ & $<0.001$ \\
\hline
\end{tabular}

SD, standard deviation; BMI, body mass index; sBMI, standardized BMI; SBP, systolic blood pressure; DBP, diastolic blood pressure; TC, total cholesterol; LDL-C, low-density lipoprotein cholesterol; HDL-C, high-density lipoprotein cholesterol; TG, triglyceride; Apo B, apolipoprotein B; HOMA-IR, homeostasis model assessment of insulin resistane. 
Table 2. Summary Table of Four PCA Rotated Component Matrix (Varimax Rotation) Models of Changes in Six Metabolic Variables Following Acute Weight loss in Obese Children and Adolescents

\begin{tabular}{|c|c|c|c|}
\hline & \multicolumn{3}{|c|}{ Factors, metabolic variable loadings, and factor variance } \\
\hline & & Obesity/blood pressure & Metabolic \\
\hline \multicolumn{4}{|l|}{ Metabolic variables entered } \\
\hline WC & WC & 0.769 & 0.371 \\
\hline BMI & BMI & 0.785 & \\
\hline TG & TG & & 0.814 \\
\hline HDL-C & HDL-C & & -0.653 \\
\hline HOMA-IR & HOMA-IR & 0.376 & 0.584 \\
\hline MAP & MAP & 0.726 & \\
\hline Variance explained $(\%)$ & & 31.3 & 26.7 \\
\hline WC & WC & 0.783 & 0.353 \\
\hline BMI & BMI & 0.772 & \\
\hline TG & TG & & 0.815 \\
\hline HDL-C & HDL-C & & -0.665 \\
\hline HOMA-IR & HOMA-IR & 0.408 & 0.559 \\
\hline SBP & SBP & 0.732 & \\
\hline Variance explained (\%) & & 31.9 & 26.5 \\
\hline WC & WC & 0.774 & 0.416 \\
\hline BMI & BMI & 0.779 & \\
\hline TG & TG & & 0.833 \\
\hline HDL-C & HDL-C & & -0.577 \\
\hline Insulin & Insulin & 0.309 & 0.676 \\
\hline MAP & MAP & 0.753 & \\
\hline Variance explained (\%) & & 30.4 & 28.4 \\
\hline WC & WC & 0.730 & 0.449 \\
\hline BMI & BMI & 0.752 & \\
\hline TG & TG & & 0.839 \\
\hline HDL-C & HDL-C & & -0.536 \\
\hline Insulin & Insulin & & 0.695 \\
\hline SBP & SBP & 0.774 & \\
\hline Variance explained (\%) & & 30.4 & 28.4 \\
\hline
\end{tabular}

Variables with factor loadings $>0.3$ are listed and variables loading on both factors are highlighted in bold.

PCA, principal component analysis; WC, waist circumference: BMI, body mass index; TG, triglyceride; HDL-C, high-density lipoprotein cholesterol; HOMA-IR, homeostasis model assessment of insulin resistance; MAP, mean arterial pressure; SBP, systolic blood pressure.

studies have typically applied EFA within cross-sectional studies of obese youths or only reported separate metabolic changes or dichotomized 'metabolic syndrome' phenotypes. ${ }^{13-16}$ Therefore, distinct features of the present study include: (1) a group of clinically obese children and adolescent participants who significantly reduced body mass and waist circumference; (2) a standardized lifestyle intervention with largely comparable dietary and physical activity modifications undertaken by all participants; (3) statistically and clinically significant reductions in several cardiometabolic variables (fasting lipoprotein-lipids, glucose, insulin, and blood pressures) not confounded by the effects of smoking, alcohol, or medications; (4) application of different EFA techniques for data reduction of cardiometabolic responses, with due consideration of variable selection and satisfactory evidence of sampling adequacy and variable communality.

Weiss et al. ${ }^{9}$ showed that $\mathrm{sBMI}$ changes were significantly and negatively correlated with changes in insulin sensitivity and positively with glucose and lipoprotein-lipid responses. Longer-term sustained weight loss also demonstrated improvement in insulin sensitivity measures and lipoproteinlipid profile responses in prepubertal obese children. ${ }^{17}$ In contrast, a retrospective case series of 207 obese children and adolescents following 12 weeks of lifestyle intervention showed that reductions in obesity measures were not asso- ciated with corresponding reductions in metabolic measures. ${ }^{18}$

Several cross-sectional studies have applied PCA to childhood and adolescent datasets, including obese cohorts, and have indicated that the 'insulin resistance syndrome' was a clinically recognizable entity with multiple pathophysiological mechanisms (reflected by between two and five underlying factors). However, studies have been inconsistent in terms of the metabolic variables considered, the precise PCA techniques employed to interpret factors, and statistical approaches adopted. Indeed, reports outlining more than two underlying factors have included as separate metabolic variables: (1) from 5 up to 16 metabolic variables simultaneously; (2) considered both arterial (SBP and DBP) blood pressure ${ }^{6}$; and (3) fasting and post oral glucose tolerance test concentrations. ${ }^{7,13,19}$ Furthermore, interpretation of factors has been complicated by consideration of TC or LDL$\mathrm{C}$ in some analyses. ${ }^{6}$

Providing clarification of the role of surrogate markers of insulin resistance (fasting insulin and HOMA) and obesity measures (raw or standardized BMI, waist circumference, and body fat content) within cardiometabolic clustering has been implicit in earlier studies. Recent cross-sectional confirmatory factor analysis findings in children and adolescents $^{19,20}$ have shown the potential usefulness of simple 
conceptual models using standard clinic-based anthropometric measures. Our findings indicated that the pattern of change in the components of the metabolic syndrome observed during an acute weight loss intervention setting can be reduced to two underlying factors by considering six clinically relevant variables to represent the components of the metabolic syndrome. Two underlying pathophysiological constructs were most consistently extracted from the metabolic intercorrelations within our longitudinal analysis incorporating up to six metabolic variables. We termed these "metabolic" and "obesity/blood pressure" factors.

Our "metabolic factor" findings are broadly consistent with several early EFA studies using orthogonal rotation which revealed five metabolic variables (ponderal index, HDL-C, TG, fasting insulin, and glucose) linked on one underlying "metabolic" factor. ${ }^{16}$ In the above analysis, a separate factor identified was characterized by both blood pressures and insulin concentration. These clustering features were reported to be independent of sex and evident within childhood (5-11 years) and adolescent (12-17 years) groups. The above investigators highlighted the key linking of a metabolic factor (hyperinsulinemia, dyslipidemia, and obesity) to hypertension through shared correlation with hyperinsulinemia/insulin resistance. Notably, two studies have undertaken PCA on metabolic clustering within selected larger cohorts of obese children and adolescents. Interestingly, Weiss et al. ${ }^{13}$ revealed three correlated factors (using an oblique rotation technique) of obesity/glucose metabolism, dyslipidemia, and blood pressure. A strong loading of insulin resistance (fasting HOMA-IR) to the obesity/glucose factor and "moderate loading" to the dyslipidemia factor was interpreted to indicate a component of insulin resistance underlying two of three factors that accounted for the majority of the variance in the dataset.

LaFortuna et al. ${ }^{7}$ showed that PCA with orthogonal varimax rotation reduced 11 correlated metabolic variables to four uncorrelated factors in boys; these included obesity/ hypertension, dyslipidemia, and insulin resistance and hyperglycemia factors. Notably, obesity and fat distribution variables also loaded significantly onto the dyslipidemia and insulin resistance factors. In obese girls, no similar commonalities were evident. However, few previous studies have examined how the various factors change together over time and neither study examined the effects of an active intervention. 6.8

Among our obese children and adolescents, combined changes in waist circumference and fasting insulin/HOMAIR appeared central to most of these underlying factors. This provides further support for the hypothesis that the different dimensions of metabolic clustering or indeed the metabolic syndrome are linked through abdominal obesity and markers of insulin resistance. Our findings of central adiposity and insulin sensitivity modulating cardiometabolic risk in obese adolescents are consistent with other investigations using different research designs and statistical approaches. ${ }^{21}$ Cross-sectional findings from the Bogalusa Heart Study have shown the use of both BMI and waist circumference for the prediction of metabolic clustering among children and adolescents has significant clinical utility.22

The results of metabolic clustering using EFA should be interpreted with some caution, given that it is not, strictly speaking, a hypothesis-testing technique. Our study indicates that important relevant information has been omitted from numerous earlier reports and several arbitrary decisions are therefore necessarily made when using this approach. Some relevant issues have previously been reviewed, $^{23}$ and our analysis adds to the conceptual understanding of this multivariate correlational technique in the context of metabolic clustering in obese children and adolescents. In our study, the number of participants is relatively small, and we were not able to run separate analyses to account for differences in metabolic responses to weight loss intervention in obese boys and girls. Furthermore, we were not able to consider the effect of differences in sexual maturity within this cohort.

In summary, despite the relatively small size of the study, we have shown very favorable cardiometabolic risk factor modifications and a distinct clustering of metabolic responses to short-term, lifestyle-induced, weight loss. Given the rapidly growing literature on the prevalence, outcomes, and underlying pathophysiology of the metabolic syndrome, we consider that our findings provide a further contribution to the understanding of this common metabolic phenotype in obese children and adolescents.

\section{Acknowledgments}

The authors would like to thank the children who participated in the study. J.P.H. and P.G. collected all data. J.P.H. and S.C. conducted all statistical analyses and wrote the manuscript. R.F.K., P.P., A.S., J.H.B., and P.G. reviewed and edited the manuscript.

\section{Author Disclosure Statement}

No competing financial interests exist.

\section{References}

1. Reaven GM. Banting lecture 1988. Role of insulin resistance in human disease. Diabetes 1988;37:1595-1607.

2. Despres JP, Lemieux I. Abdominal obesity and metabolic syndrome. Nature;444:881-887.

3. Cook S, Weitzman M, Auinger $P$ et al. Prevalence of a metabolic syndrome phenotype in adolescents: Findings from the third National Health and Nutrition Examination Survey, 1988-1994. Arch Pediatr Adolesc Med 2003;157: 821-827.

4. Brambilla P, Lissau I, Flodmark CE et al. Metabolic riskfactor clustering estimation in children: to draw a line across pediatric metabolic syndrome. Int J Obes (Lond) 2007;31: 591-600.

5. Anderson PJ, Critchley JA, Chan JC et al. Factor analysis of the metabolic syndrome: obesity vs insulin resistance as the central abnormality. Int J Obes Relat Metab Disord 2001;25: $1782-1788$

6. Goodman E, Daniels SR, Meigs JB et al. Instability in the diagnosis of metabolic syndrome in adolescents. Circulation 2007;115:2316-2322.

7. Lafortuna CL, Adorni F, Agosti F et al. Factor analysis of metabolic syndrome components in severely obese girls and boys. J Endocrinol Invest 2009;32:552-558.

8. Maison $\mathrm{P}$, Byrne $\mathrm{CD}$, Hales $\mathrm{CN}$ et al. Do different dimensions of the metabolic syndrome change together over time? Evidence supporting obesity as the central feature. Diabetes Care 2001;24:1758-1763.

9. Weiss $R$, Shaw $M$, Savoye $M$ et al. Obesity dynamics and cardiovascular risk factor stability in obese adolescents. $\mathrm{Pe}$ diatr Diabetes 2009;10:360-367. 
10. Cole T], Bellizzi MC, Flegal KM et al. Establishing a standard definition for child overweight and obesity worldwide: international survey. BMJ 2000;320:1240-1243.

11. Gately PJ, Cooke CB, Barth JH et al. Children's residential weight-loss programs can work: A prospective cohort study of short-term outcomes for overweight and obese children. Pediatrics 2005;116:73-77.

12. Lawlor DA, Ebrahim S, May M et al. (Mis)use of factor analysis in the study of insulin resistance syndrome. Am J Epidemiol 2004;159:1013-1018.

13. Weiss R, Dziura J, Burgert TS et al. Obesity and the metabolic syndrome in children and adolescents. $N$ Engl $J$ Med 2004;350:2362-2374.

14. Kelishadi R, Ardalan G, Adeli $\mathrm{K}$ et al. Factor analysis of cardiovascular risk clustering in pediatric metabolic syndrome: CASPIAN study. Ann Nutr Metab 2007;51:208-215.

15. Lambert M, Paradis G, O'Loughlin J et al. Insulin resistance syndrome in a representative sample of children and adolescents from Quebec, Canada. Int J Obes Relat Metab Disord 2004;28:833-841.

16. Chen W, Srinivasan SR, Elkasabany A et al. Cardiovascular risk factors clustering features of insulin resistance syndrome (Syndrome X) in a biracial (Black-White) population of children, adolescents, and young adults: The Bogalusa Heart Study. Am J Epidemiol 1999;150:667-674.

17. Martos-Moreno GA, Barrios V, Martinez G et al. Effect of weight loss on high-molecular weight adiponectin in obese children. Obesity (Silver Spring) 2010;18:2288-2294.

18. Shalitin S, Yackobovitch-Gavan M, Phillip M. Prevalence of thyroid dysfunction in obese children and adolescents before and after weight reduction and its relation to other metabolic parameters. Horm Res 2009;71:155-161.
19. Li C, Ford ES. Is there a single underlying factor for the metabolic syndrome in adolescents? A confirmatory factor analysis. Diabetes Care 2007;30:1556-1561.

20. Martinez-Vizcaino V, Martinez MS, Aguilar FS et al. Validity of a single-factor model underlying the metabolic syndrome in children: A confirmatory factor analysis. Diabetes Care 2010; 33:1370-1372.

21. Bacha F, Saad R, Gungor N et al. Are obesity-related metabolic risk factors modulated by the degree of insulin resistance in adolescents? Diabetes Care 2006;29:1599-1604.

22. Katzmarzyk PT, Srinivasan SR, Chen $W$ et al. Body mass index, waist circumference, and clustering of cardiovascular disease risk factors in a biracial sample of children and adolescents. Pediatrics 2004;114:e198-e205.

23. Meigs JB. Invited commentary: Insulin resistance syndrome? Syndrome X? Multiple metabolic syndrome? A syndrome at all? Factor analysis reveals patterns in the fabric of correlated metabolic risk factors. Am J Epidemiol 2000;152:908-911.

Address correspondence to: James P. Hobkirk, B.Sc.

Department of Academic Cardiology

Postgraduate Medical Institute

Medical Research, Teaching \& Day Surgery (Daisy Building) Castle Hill Hospital Castle Road

Cottingham, Kingston upon Hull HU16 5JQ United Kingdom

E-mail: james.hobkirk@hey.nhs.uk 